\title{
Las Ciencias Sociales en América Latina: lo permanente y transitorio, preguntas y desafíos de ayer y hoy
}

\author{
Dimas Floriani \\ Profesor Titular en los programas de posgrado en Medio Ambiente y Desarrollo y \\ en Sociología de la Universidad Federal de Paraná (UFPR). \\ Email: floriani@ufpr.br
}

\begin{abstract}
Resumen: El artículo intenta contestar a dos preguntas centrales acerca del rol de las ciencias sociales latinoamericanas: ¿Cuáles son los principales desafíos que enfrentarán las Ciencias Sociales en nuestro continente? ¿Cuáles son las principales preguntas que deberemos hacernos para contribuir al futuro de la democracia, de la paz y de la sustentabilidad en nuestras sociedades? Para ello, buscamos hacer un balance histórico y epistemológico de las ciencias sociales desde mediados del siglo XX, sus distintos métodos y matrices teóricas. A la vez se presentan otros temas emergentes y estrategias plurales basadas en alternativas metodológicas y nuevos compromisos éticos y políticos.

Palabras clave: Desafíos de las ciencias sociales; América Latina y las ciencias sociales; alternativas teóricas y metodológicas de las ciencias sociales; compromisos de la teoría con la política y la ética.
\end{abstract}

\section{Social Sciences in Latin America: the permanent and transient, questions and challenges of yesterday and today}

\begin{abstract}
The article attempts to answer two central questions about the role of Latin American social sciences: What are the main challenges facing Social Sciences in our continent? What are the main questions that we should make ourselves to contribute to the future of democracy, peace and sustainability in our societies? To this end, we seek to make an historical and an epistemological theoretical balance of social sciences since the mid-twentieth century, their different methods and theoretical matrices. In turn, other emerging issues and plural strategies based on new methodological and ethical and political commitments alternatives are presented.

Key words: Challenges of social sciences; Latin America and the social sciences; theoretical and methodological alternatives of the social sciences; commitments of the theoryto politics and ethics.
\end{abstract}

\section{Ciências Sociais na América Latina: o permanente etransitório, questões e desafios de ontem e de hoje}

Resumo: O artigo tenta responder a duas questões centrais sobre o papel das ciências sociais latino-americanas: Quais são os principais desafios que enfrentarão as Ciências Sociais em nosso continente? Quais são as principais questões que nós deveremos fazer para contribuir ao futuro da democracia, da paz 
e da sustentabilidade em nossas sociedades? Para este fim, buscamos fazer um balanço histórico e epistemológico das ciências sociais desde meados do século XX, os seus diferentes métodos e matrizes teóricas. Simultaneamente se apresentam outros temas emergentes e estratégias plurais com base em alternativas metodológicas e novos compromissos éticos e políticos.

Palavras-chave: Desafios das ciências sociais; América Latina e as ciências sociais; alternativas teóricas e metodológicas das ciências sociais; compromissos dateoria com a política e a ética.

$$
* * *
$$

\section{Introducción}

Las preguntas acerca de los desafíos de las ciencias sociales en nuestro continente tienen implícito un conjunto de cuestiones, sin las cuales es difícil armar algunas respuestas pertinentes. La primera cuestión se refiere a los aspectos estructurales (históricos y epistemológicos) en los cuales se enmarca la producción misma del conocimiento académico de las ciencias sociales.

Por aspecto histórico estructural uno debe entender las dimensiones societales en las cuales transcurren los fenómenos sociopolíticos, económicos, intelectuales, culturales, ambientales, tecnológicos... y que se suceden de manera diacrónica y heterogénea en nuestras formaciones sociales latinoamericanas. La manera de como uno se representa esta dinámica corresponde a diferentes formas de traducir los distintos entendimientos y disputas de sentidos por los usos y modas teóricas y autorales que se reproducen, instituyen y legitiman en las distintas agencias de producción del conocimiento o en la manera de como ocurren las discusiones públicas y en las comunidades o corporaciones académicas, por los medios de comunicación, las redes sociales de internet, etc.

Sin embargo, las modas intelectuales corresponden a eventos más o menos estables, de corta, mediana y larga duración pero que pueden cambiar radicalmente de acuerdo a la pérdida de interés o cuando son deslegitimados y desvalorizados por los efectos ideológicos producidos por la pérdida de interés o cuando los temas sufren un importante déficit mediático e incredulidad y depreciación; hay una especie de bolsa de valores simbólicos que mide lo que más está en boga de autores y temas que empiezan a ganar o perder fuerza según las circunstancias. Después de todo, recordemos lo que nos dice Edgar Morin (1984) acerca de la producción intelectual: las teorías también son biodegradables, nacen y mueren o resurgen ya con distintos matices según los contextos intelectuales, políticos y culturales en que se generan.

Si uno se acuerda de lo que nos decía Marx en sus Manuscritos Económico Filosóficos (1972, p. ) acerca de la relación entre pensamiento y realidad (lo que incluye la acción), que son dos entes distintos pero que en algún momento se conectan, así ocurre también con la producción del co- 
nocimiento por autores y agencias que siguen su ruta y temporalidad pero que en algún momento también se conectan (positiva o negativamente) con el contexto en el cual se producen los eventos intelectuales, una especie de autopoiesis (Maturana y Varela, 1996) que puede seguir su curso pero que después es molestada por la "deriva” o el clinamen que Lucrécio, en conformidad con las ideas epicuristas originales, hablaba de desvío imprevisible (o estocástico) que ocurría "en ningún lugar o tiempo fijo" cuando hablaba de los átomos.

Así son los eventos y circunstancias intelectuales que se reproducen, se propagan, se contienen y se transforman bajo entradas e impulsos imprevisibles pero que después siguen su curso normal hasta que sufren nuevos remezones. En este sentido, este movimiento o dinámica de las ideas en su lugar o fuera de él se lleva a cabo en el interior de instituciones que operan como guardianes de verdades transitorias, pero muy efectivas mientras funcionan para luego caer en descrédito cuando ya no funcionan, por fuerza de las externalidades socioculturales y políticas.

\section{Una visión panorámica de las ciencias sociales latinoamericanas desde la mitad del siglo XX en adelante}

Si logramos localizar este tipo de sucesos relativos a la producción de ideas y de conocimientos al interior de las ciencias sociales en el contexto latinoamericano de las últimas décadas, es posible verificar la permanencia y la transitoriedad de un conjunto de temas y debates que impregnaban las agencias de conocimiento en América Latina.

Mismo que algunos de estos temas perdieron fuerza o fueron remplazados por otros, no se puede quitarles fuerza y pertinencia en su tiempo, pues se remitían a un complejo de condiciones intelectuales, culturales, políticas, económicas, epistemológicas... en aquel período.

Al vislumbrar algunos temas que se establecieron desde los años 1950 en nuestro continente, se nota que son marcados por los desafíos del desarrollo nacional, los problemas de planificación y gestión política de los estados nacionales, la industrialización y la transformación de las condiciones sociales y relaciones de producción en el agro, los conflictos políticos derivados de un cuadro de profunda desigualdad social, en un panorama de baja institucionalidad democrática, por la dificultad de acceso de los sectores populares a un marco institucional fuertemente controlado por las oligarquías políticas... Son añadidos a estos factores las distintas teorías de la modernización que partían de presupuestos opuestos: por un lado las teorías que pretendían repetir las etapas de crecimiento económico del norte hacia el sur - como la obra de Rostow (1959) y de Lipset (1959) y por el otro, desde el sur decretaban un grito de guerra teórico e ideológico, en contra de un arreglo reformista, a raíz del modelo capitalista periférico y dependiente en crisis ( en especial los trabajos de los críti- 
cos de la teoría de la dependencia, como Marini(1969, 1973), dos Santos(1972 ) y Frank(1969) ${ }^{1}$.

Imposible de pensar lo que se gestaba teóricamente en las ciencias sociales sobre los desafíos enfrentados por las sociedades latinoamericanas, al margen de ese período y de su contexto internacional. Los marcos de referencia teórico-metodológicos de las ciencias sociales tenían un fuerte arraigo en las teorías estructural-funcionalistas y marxistas y el telón de fondo o el espejismo de estas teorías reflejaba imaginarios acerca de la misión y los destinos de las sociedades nórdicas y por lo general con actores sociales (clases, sindicatos, instituciones, estado, etc.) ya definidos de antemano por los modelos teóricos elaborados en contextos europeos o norte-americanos, en favor o en contra de estos modelos hegemónicos.

Por supuesto que la situación de ese período que varía del 1950 al 1970 era más compleja y rica y su simplificación puede enmascarar o caricaturizar la riqueza de la época. Hay que hacer justicia a instituciones y autores originales que irradiaban sus ideas y obras desde la creación de la CEPAL y una serie de intelectuales que dejaron su legado como un marco de referencia para la posteridad en todos los países del continente y que influenciaron a un gran número de investigadores y militantes de la época ${ }^{2}$.

Empero, la señal de los tiempos se hace notar con las crisis y rupturas que se siguen a los años 1970 y que inauguran los modelos autoritarios por las dictaduras militares, particularmente en el cono sur. En función de las restricciones a la libertad de crítica contra aquellos regímenes políticos, emergen investigadores norte-americanos ( Stepan, Skidmore...) y europeos (Touraine, Rouquié...) que se especializan en teorías del autoritarismo y que en conjunto con algunos intelectuales latinoamericanos influencian en especial a politólogos argentinos, bolivianos, brasileros, chilenos, colombianos, mexicanos, peruanos, (Cardoso, Hinkelammert, Calderón, Garretón, Lechner, O’Donnel, Laclau, Weffort, Stavenhagen, Gonzalez Casanova, Quijano, Ianni...).

Las ciencias sociales son desplazadas de su papel anterior, cuando cuadros intelectuales se reunieron con algunos gobiernos que pensaban las estrategias de desarrollo desde el aparato del Estado nacional. No es que esto no hubiese ocurrido con los gobiernos militares que fueron asesorados por economistas formados en academias de Estados Unidos que se rigen por la cartilla de las teorías monetaristas o neo clásicas, como la escuela de Chicago que jugó un papel emblemático en Chile, con los ahijados de Milton Friedman. Sin embargo, las teorías del desarrollo social fueron remplazadas por las teorías económicas del crecimiento que lanzan las bases para la proliferación del pensamiento neoliberal subordinando las instancias de la organización social a la racionalidad y funcionamiento del mercado.

De las ventajas comparativas se pasa a las ventajas competitivas. El Estado se encarga de la seguridad, poco de salud y de educación y será el 
mercado el que define los rumbos y los rubros del intercambio de bienes y de sus exportaciones e importaciones. Poco importa si la clase media desarrolla valores democráticos como pretendían los teóricos de la modernización y de la democracia en los años 50 y 60; lo que importa es que aquella se inscriba en una agenda de modernidad consumista; la cultura cívica debe ser la de las estrategias individuales de la meritocracia y de la privatización de los bienes esenciales, como la formación profesional, los planes de salud y la previdencia social. Los servicios públicos entran en una etapa de precarización y de descrédito.

A partir de esto, se establecieron otras bases para la introducción de nuevos temas de debate e investigación, ahora alineados con el surgimiento de nuevas inquietudes intelectuales, en todo el mundo. Es como si el mundo se hubiese reunificado, con el centro en el norte, después de la caída del socialismo realmente existente. El hito para este tránsito fueron las políticas económicas de Reagan y de Thatcher que van desaguar en el Consenso de Washington. Según David Harvey $(2005,2010)$ la política global neoliberal es un sistema que beneficia pocos a las expensas de muchos y tiene como resultado la resignificación de la distinción de clase por medio de la ‘acumulación por desposesión'. Sería un error conceptual y político concebir el capitalismo desde un punto de vista de la ética y de la regulación, pues su esencia es regida por una amoralidad y una ausencia de ley.

Las teorías de la globalización inauguran en los años 1990 una nueva ideología en el marco de las investigaciones académicas y ganan aras de popularización en los medios de comunicación; no importa si uno piensa el mundo desde Burundi, Sri Lanka, Paraguay, Inglaterra, Estados Unidos o Francia. El mundo es "uno", "igual” en cualquier parte; lo que cambia (además de las genéricas referencias a lo global y a lo local) son los distintos temas de investigación, un sin número de objetos, que pueden ser interpretados con referencia a autores universales, legitimados en las academias de todos los lugares, traducidos en todos los idiomas y que tienen explicaciones universales.

El mundo se hizo chico, no solo por las tecnologías pero también por los investigadores que se desplazan a todas las universidades del planeta. Las teorías y epistemologías que dan soporte al nuevo pensamiento son las llamadas teorías pos modernas. Las ciencias sociales reciben inspiraciones de otras áreas de divulgación y popularización. Escritores como Bauman son leídos en todo el mundo, en diversos temas sociológicos. Sin juicios de valor anticipados, uno puede sin embargo valorar este fenómeno como positivo en muchos casos, una vez que se desplaza al gran público lecturas y abordajes que se restringían antes a un público especializado. Por otro lado, tecnologías más accesibles permiten que se consulten obras digitalizadas que antes solo era posible encontrarlas en papel, muy costosas y en idioma original, además de acceso fácil en entrevistas de especialistas disponibles por internet, en distintos idiomas pero principalmente en inglés. 
Es bien verdad que la europeización de los marcos teóricos siempre estuvo presente en los centros de pensamiento latinoamericano ${ }^{3}$, por razones coloniales y que autores autóctonos intentaban oponerse, aprovechando la ola de la contestación en contra de la colonialidad del saber y del poder (Quijano, Mignolo, etc.). Las raíces de esta contestación se ubican en el mismo período en que se gestaban las teorías de la dependencia o de la resistencia al colonialismo e imperialismo (Fanon, Amin, Casanova y otros pensadores $)^{4}$.

Todavía aquél movimiento se mostraba frágil y no tenía mucho arraigo en los centros de investigación de prestigio, con salvedad de algunos íconos del pensamiento, (Gutiérrez, Boff, Dussel, Josué de Castro...); además de la acción de agencias morales en los sectores de base de la iglesia católica, con la teología y filosofía de la liberación. En contraste, en los años pos 1990 es posible localizar grupos de investigadores que se proponen a sistematizar y divulgar su producción en distintas universidades del globo, bajo la rúbrica del pensamiento pos colonial (de Sousa Santos, Said, Mignolo, Quijano...) a pesar de que se inscriben todavía como minoritarios frente al gran mainstream que enuncia sus proyectos investigativos de acuerdo a autores universales, los clásicos modernos y contemporáneos, leídos y citados en todas las academias del mundo, con una devoción irrefutable, aceptados como teóricos passe-partout, obligados por las referencias bibliográficas en las monografías y tesis de formación de los alumnos. Así, cada corriente de pensamiento en las distintas disciplinas de las ciencias sociales tiene sus referencias clásicas, modernas y contemporáneas, en su gran parte ubicados en el norte ${ }^{5}$.

La profusión de temas y obras de estos especialistas universales pueden ser identificados en las publicaciones de periódicos, en los centros de investigación, de los numerosos congresos y seminarios que se multiplican a cada año y en todos los lugares.

\section{Una breve mirada a las agencias de conocimiento latinoamericanas: ejes temáticos y líneas de investigación}

Está claro que sería presuntuoso querer abordar en este artículo toda la producción intelectual de las ciencias sociales en América Latina. Para ello sería necesario, al menos, crear un grupo de investigación que durante mucho tiempo pudiese hacer una encuesta de universidades, departamentos y cursos, así como las principales revistas en que se publican las investigaciones.

Para que se pudiera tener un panorama más cercano de las transformaciones ocurridas en los últimos 30 años en los procesos de organización de las agencias de conocimiento (universidades y centros de investigación latinoamericanos en el área de humanidades y ciencias sociales) sería fundamental dibujar el incremento numérico y cualitativo de las áreas y líneas 
de investigación de los postgrados en el continente, los periódicos académicos y su acreditación en los principales indexadores de calidad. Es notorio en el caso de Brasil la ampliación de los postgrados a todas las regiones del país. Históricamente, los postgrados de humanidades y ciencias sociales se localizaban fundamentalmente en 6 estados (São Paulo, Rio de Janeiro, Minas Gerais, Pernambuco y Rio Grande do Sul). A raíz de esta expansión, se fortalecen las asociaciones de postgrado en todas las disciplinas de humanidades y de ciencias sociales. El reflejo de esta expansión se hace notar en las asociaciones latinoamericanas, tales como ALAS (de Sociología), ALA (Antropología) que realizan periódicamente sus congresos ${ }^{6}$.

Las asociaciones y congresos de investigadores y educadores universitarios reflejan en gran medida los temas que emergen del debate abierto, desde las organizaciones y movimientos sociales. Como hipótesis, es posible lanzar la siguiente pregunta respecto a la institucionalidad de la investigación y sus vínculos con el debate político abierto con los sectores sociales: en la medida que las instituciones de investigación y enseñanza se abren al diálogo y a las prácticas democráticas, hacia una ciencia no corporativa en sus distintas formas de producción, difusión y discusión de sus temas de investigación y de proyectos comunitarios, es probable que se incorporen con más agilidad los nuevos temas que emergen de los conflictos sociales.

Sin juicio de valor, habría que comparar las principales instituciones nacionales e internacionales latinoamericanas de investigación y enseñanza, desde sus distintas formas de organización de equipos temáticos para percibir en que tiempo ocurren las nuevas incorporaciones temáticas y el grado de apertura para nuevos temas. Como ejemplo, las instituciones que tienen fuerte grado de institucionalidad como CEPAL, FLACSO, PNUMA se diferencian de las asociaciones académicas, programas de postgrado, organizaciones no gubernamentales o comunitarias, etc. Al mismo tiempo, averiguar cómo se produce conocimiento, de forma tradicional o fuertemente disciplinaria o con otras metodologías de investigación (interdisciplinaridad, transdisciplinaridad).

En el dominio de las ciencias sociales es posible decir que la tradición europea de ciencia inaugurada en el siglo XIX ha lanzado la distinción entre ciencias naturales y sociales, o sea la gran división de las dos culturas (Snow, 1987) ${ }^{7}$ anticipada por la discusión mantenida por los neokantianos y explicitada por Max Weber (1965) entre las ciencias nomotéticas, que buscan leyes para establecer las regularidades de los fenómenos observados y aquellos otros fenómenos cargados de significados culturales designados como fenómenos ideográficos. Sin embargo, Weber busca un tratamiento específico para los fenómenos histórico-culturales empleando procedimientos metodológicos que por similitud se asemejan a los modelos nomotéticos (los tipos ideales); lo que sería definitivo en la distinción entre las dos culturas, según Weber, es que los fenómenos histórico-culturales son singulares y que no se repiten de la misma manera en todas las partes. 
Mismo que sea posible contestar esta visión, sea del punto de visto epistemológico o del punto de vista ideológico, pues Weber buscaba legitimar el modelo europeo y occidental como lo único capaz de desarrollar el moderno sistema capitalista; el método comparativo que utiliza desde la introducción de la Ética Protestante y el Espíritu del Capitalismo (2001) es una prueba de como rescata la especificidad del cambio en la racionalidad del ethos en el trabajo, por la relectura que hace Lutero acerca del concepto de profesión, resignificada desde la semántica religiosa que mesclaba aspectos confesionales con aspectos seculares (la relación entre la noción de vocación con la de profesión).

Marcuse (1972) demuestra que las ciencias sociales nacen en Europa desde dos vertientes: de la filosofía (Marx y Weber se inscriben en esta tradición, con dos referentes centrales: Hegel y Kant) y del desarrollo mismo de las ciencias en el siglo XIX que trataban de establecer una cartografía de las áreas y disciplinas, con sus objetos específicos, sus teorías y métodos observacionales. En gran medida, Durkheim se inscribe en este segundo modelo cuando reivindica para la sociología un objeto (los hechos sociales) y un método basado en la objetividad y coercitividad de los mismos, a ejemplo de los fenómenos naturales que existen independientemente de la voluntad o subjetividad del observador. Durkheim intenta acortar así la división entre, por un lado, la epistemología de las leyes naturales y, por otro, los fenómenos sociales que a pesar de sui generis, pueden ser interpretados bajo el mismo método objetivo de las demás ciencias; sin embargo a igual método corresponden distintos conceptos; lo que alejaría según esta lógica, la posibilidad de un diálogo entre las ciencias, una vez que los fenómenos de la naturaleza y los fenómenos socioculturales son ontológicamente distintos.

De estos tres clásicos de las ciencias sociales modernas, Marx es el único que no se separa de la naturaleza pues la misma es inseparable de las formas de apropiación y transformación de la materia por el trabajo humano. Las consecuencias de este modelo analítico no exenta a Marx y a sus seguidores de subrayar la importancia del desarrollo científico y del progreso tecnológico que pueden liberar la humanidad de sus límites y de la explotación del trabajo sin medir los efectos que ello tendría sobre los límites mismos de los recursos naturales, sus biomas y ecosistemas. John Bellamy Foster (2005) intenta demostrar lo contrario en la formación del pensamiento marxiano.

En un trabajo colectivo de mediados de 1990 organizado por Immanuel Wallerstein (1996) hay un intento de explicar cómo se han portado las ciencias sociales en los últimos 200 años y la quiebra reciente de paradigmas en sus procedimientos, sea en término de elección de sus temas de investigación o en las nuevas metodologías que se ven obligadas a adoptar, en un sentido opuesto a lo que las caracterizaba, es decir, cada una de ellas profundizando sus múltiples temas pero aislándose de las demás disciplinas.

Así, los años 1990 son también los de mea-culpa para las ciencias sociales atrapadas por la súper especialización y sus aislamientos progresi- 
vos. Giddens y Turner (1999) en uno de sus trabajos indican la crisis en que se ven envueltas las ciencias sociales que se espejaban en las ciencias naturales como ejemplo; en el momento en que se afirmaban desde aquel modelo, las ciencias sociales se vuelven epistemológicamente huérfanas cuando irrumpe la crisis en la física que se despide de un modelo unitario sobre lo que se entiende por ciencia y por objetividad, imponiendo a algunos científicos una nueva agenda y una nueva alianza entre sociedad y naturaleza (Prigogine y Stengers, 1980).

En general se puede decir que las ciencias sociales en América Latina, en buena parte del tiempo desde los años 1950 en adelante, con las rupturas y las transiciones democráticas, se volvieron rehenes de concepciones de modernización trunca, por cuenta del subdesarrollo estructural dominado por rasgos tradicionalistas y no típicamente capitalistas, distintamente de algunas sociedades centrales tomadas como ejemplo de sociedades más desarrolladas (Barrington Moore, Jr. 1966).

$\mathrm{Al}$ revés, lecturas más recientes, mismo que partiendo de referentes histórico-estructurales críticos del siglo pasado, parecen superar el marco analítico de las teorías dicotómicas de la modernización, introduciendo categorías más sofisticadas que reivindican la especificidad cultural, política e histórica de las sociedades latinoamericanas.

Así, los estudios que parten de la crítica a la colonialidad del poder y del saber reconocen que la modernidad occidental no sería posible sin que América Latina estuviese presente en el escenario de la conquista y que introduce otro tipo de modernidad. (Martucelli,2010). En esta perspectiva, las sociedades latinoamericanas no son modernas pero tampoco son sociedades tradicionales ${ }^{8}$.

Partiendo de otro punto de referencia de la sociología del subdesarrollo y de la dependencia, la perspectiva poscolonial rompe con la imagen binaria - nacionalista o indigenista - no adscrita a una visión holística de explicación social, en benefício de lecturas que subrayan la complejidad de las fronteras culturales y políticas (Martucelli, 2010, p. 41). En este sentido, la pareja modernidad-colonialidad son las dos caras de una misma moneda.

Como se ha notado por las exposiciones anteriores, encontramos dos tipos de modelos teóricos de las ciencias sociales de interpretación de los procesos históricos de la formación social latinoamericana, en los últimos 50 años: de un lado, una interpretación holista o estructural acerca de las concepciones truncas o difíciles del desarrollo capitalista, con énfasis en los aspectos económicos y políticos estrechamente vinculados y subordinados al capitalismo central.

Esta interpretación, en sus distintas vertientes, estructural funcionalistas o marxistas, partió de modelos universales, con categorías teóricas aplicables a todo tipo de desarrollo histórico-social y cultural, a pesar de la teoría de la dependencia reivindicar una especificidad teórica 
para la periferia latinoamericana. Desde otra perspectiva, una matriz teórica que hace hincapié en los estudios poscoloniales y culturales y que no necesita partir de la oposición entre modernidad y tradición, prefiriendo considerar la situación histórica latinoamericana formada de modernidades en el plural, a la vez híbridas y heterogéneas.

En esta última dirección se encuentra el estudio realizado sobre cohesión social y democracia en América Latina, por Bernardo Sorj y Danilo Martuccelli (2008): en sus 4 partes, el libro contempla las transformaciones del lazo social; los actores colectivos y formas de representación; problemas y promesas: economía informal, crimen y corrupción, normas y derechos; Estado, Nación y política(s) en el despertar del siglo XXI.

De una manera muy somera, se puede afirmar que esta interpretación se ubica en el marco teórico que, además de reivindicar una especificidad histórico-cultural de las formaciones sociales latino-americanas, se inscribe en una hermenéutica pos colonial, pero sin el éxtasis de los trabajos que todavía reivindican rasgos utópicos de superación de la subalternidad, con raíces en Fanon (2002), hacia procesos de autonomización y de emancipación inspirados en las propuestas más recientes de los movimientos sociales, como la formación del Forum Social Mundial, la reemergencia de los pueblos originarios ( Bengoa, 2002) y de su filosofía del Buen Vivir (Acosta, De la Cuadra, 20015 ) y la organización de nuevas formas de superación de la pobreza y de la marginación social, como la economía solidaria, el cooperativismo y la lucha por la tierra y la reforma agraria (De Sousa Santos, 2010).

Una de las principales tesis del libro de Sorj y Martuccelli tiene a ver con su adhesión voluntaria al paradigma de la indeterminación o incertidumbre respecto a los procesos de democratización, por ejemplo, que se guiaban antes por identidades de clase, de género y étnicas y que ahora se encuentran obstruidos por la fuerte individuación de los procesos sociales que reverberan en la poca eficacia de los sistemas políticos en acoger las demandas colectivas, una vez que las iniciativas sociales se dan al margen (exit) o fuera del sistema político, en contra del espacio público (delincuencia, violencia urbana, o migración al extranjero) ${ }^{9}$.

Hasta aquí hemos encontrado voces disonantes acerca de los modelos interpretativos de América Latina, desde las obras inaugurales de la mitad del siglo XX hasta ahora. En lo que respecta a los autores que hemos traído a título de colación, se puede afirmar que encontramos las siguientes oposiciones hermenéuticas: los autores críticos de la dependencia basaban sus interpretaciones en los factores histórico estructurales, en especial en los factores determinantes de la economía (dinámicas de la división internacional del trabajo y de modernización del mercado), de la política (los sistemas de gobierno con centralidad en los Estados nacionales) y geopolíticos (subordinación a las metrópolis o a los imperios).

Los autores que tienen sus referentes en las teorías de la pos colonialidad han formulado sus modelos interpretativos en fenómenos no 
fundamentalmente económicos o políticos (más bien de áreas de frontera en que se combinan aspectos objetivos y subjetivos, fenómenos culturales, psicosociales, étnicos, interculturales, de género, de sexualidad...), en la búsqueda de otros referentes teóricos diferentes de aquellos basados en autores con matrices nórdicas que pensaban desde sus matrices los destinos para las demás sociedades.

Sin embargo, de la misma manera que los análisis estructurales o holistas tienen variantes, lo mismo se puede decir de los estudios con matrices pos coloniales, es decir, mientras algunos estudios rescatan la tradición de las dinámicas colectivas, otros las rechazan, para subrayar que las estrategias de los actores son influenciados no solamente por procesos de resistencia (voice y loyalty) pero igualmente por los desvíos provocados por la ideología y la cultura de la individuación capitalista (exit).

\section{La pregunta por una ciencia pública y pertinente y por una ética en la investigación}

De una manera u otra, las actividades académico-científicas mantienen vínculos con el entorno social y son institucionalizadas de diversas formas. No es esta cuestión genérica que nos interesa en este momento, sino como se generan los mecanismos de la producción social del conocimiento acerca de los usos de la ciencia, las motivaciones y razones que conducen que determinada corporación académica elija tal o cual tema de investigación y cómo las instituciones públicas de gestión de las agencias de conocimiento valoran determinados temas en desmedro de otros.

John Ziman (2002), científico y filósofo de la ciencia inglés nos habla de una ciencia pos académica en el sentido de que los procedimientos del quehacer científico no se limitan exclusivamente a la cúpula en la que están situados los científicos pero también a un conjunto de acciones complejas que median los sistemas internos de las corporaciones y grupos de investigación, los actores públicos y privados que gestionan, controlan y evalúan los modos de funcionamiento científico y fomentan las inversiones en investigación y desarrollo.

Además, aquellas acciones complejas en las cuales se entretejen y confluyen factores de distintos órdenes tales como concepciones de ciencia, investigaciones encargadas y que resultan aplicables en distintos campos de la tecnología y de los usos sociales, tales como tecnologías digitales de comunicación, producción y consumo de fármacos, aplicaciones químicas al agro y a los géneros alimentares, encuestas sociales, estudios e informes sobre comportamiento humano, e incontables otras áreas en que se aplican los conocimientos y las investigaciones realizadas en laboratorios.

La producción del conocimiento en las sociedades actuales se instituye en agencias académicas y en otras entidades de aplicación e innova- 
ción técnica. Desde estos sistemas de funcionamiento, gestión y administración del conocimiento emergen distintas concepciones de ciencia, a la vez que se gestan críticas epistemológicas, políticas y culturales a sus usos, de parte de distintos actores sociales subalternos que no se ven contemplados por tales sistemas cognitivos.

La tradición derivada de la historia de las ciencias (ciencias normales) es instaurada por una convención, (una especie de Parlamento de las Ciencias) que a ejemplo de las instancias de legitimación de una o de múltiples autoridades políticas depende de un sistema de evaluación, control y configuración de instituciones académico-científicas. Bourdieu (2002) presenta la posibilidad de interpretar el sistema científico y sus prácticas con la idea de campo con escisiones entre sus participantes, autoridades y conflictos en la distribución de los recursos para la investigación, lo que produce una estratificación social del sistema académico-científico.

Una sociedad formada por clases sociales puede comportar espacios públicos y privados de producción y reproducción del sistema de las ciencias. Este orden es regulado por los Estados Nacionales y sus interconexiones internacionales; por las instituciones y carreras de los científicos y sus vinculaciones a un sistema de jerarquías en la distribución de los bienes educacionales, definidos por la estructura de clases que controlan los capitales sociales cuyo sistema de ingresos condiciona la capacidad de cada persona ingresar al sistema educativo.

La organización del sistema académico-científico se puede vislumbrar desde algunas interacciones entre las dimensiones epistemológicas, institucionales, y político-culturales acerca del significado del conocimiento científico, de sus usos y sus aplicaciones.

En el aspecto epistemológico nos enfrentamos con lo que cada disciplina científica reivindica para si un modelo ideal de ciencia, además de lecturas que se hacen de la historia de la ciencia y las prácticas de investigación y enseñanza impartidas. Piaget (1967) identifica que hay distintas epistemologías: las científicas, que derivan de la ciencia misma; las extracientíficas (de los enfrentamientos filosóficos); las meta-científicas (derivadas de valores, creencias, religión, arte, crítica social, etc.).

Por dimensiones institucionales se entienden las formas de cómo se organizan las cartografías de las ciencias; la organización en forma de sectores, departamentos, institutos, centros de investigación; los tipos de posgrados y las asociaciones establecidas de investigación; la cultura científica generada por esta distribución y la crítica que se hace al interior de la comunidad científica sobre la gestión, el control, el sistema de evaluación de los pares; las nuevas asociaciones que derivan de esta crítica y los nuevos reordenamientos de las cartografías regionales de las disciplinas.

Dentro del marco de estos enfrentamientos se encuentran algunos de los conflictos entre concepciones de la ciencia normal, con la historia y 
los métodos de las disciplinas modernas y la ciencia pos-normal o posacadémica o aún nómada que presenta una estrategia híbrida entre distintos saberes científicos y no científicos o culturales, como es el caso de algunas metodologías interdisciplinarias y transdisciplinarias.

Hay que subrayar que una concepción alternativa de ciencia depende de nuevos arreglos epistemológicos para dar soporte a nuevas afiliaciones científicas, por ejemplo, la apelación a teorías de la complejidad, del holismo sistémico, de la coevolución, etc.

En el caso de algunos de los estudios transversales o temas de frontera como los ambientales (saberes y prácticas en los usos de los recursos naturales), educacionales (en comunidades tradicionales), medicales (usos de medicinas alternativas) o lingüísticos o interculturales (diálogos entre distintas culturas) el desafío está en observar como cada uno de estos nuevos procedimientos es incorporado o rechazado por las instituciones académicas, en el pregrado o posgrado.

Resulta que se establece una determinada cultura académica según cómo son tratados los métodos de investigación, la enseñanza y la aplicación de los conocimientos en determinados contextos sociales. La cultura académica se deriva de las prácticas asentadas en las concepciones de la ciencia, más allá de los sistemas de control, evaluación, bono o sanciones ejercidos institucionalmente. Hay por así decir un acuerdo tácito y explícito entre concepciones de la ciencia y las prácticas de investigadores y enseñantes que refuerzan o no la cultura institucional académica.

De esta manera, los sistemas académicos de producción de conocimiento se enfrentan a una trama organizativa y compiten espacios y sentidos con las siguientes características: por un lado, los avatares del mercado y una ciencia privada y por otro, interrogantes y conflictos de valores acerca de una ciencia pública (una ciencia ¿para quienes, con quienes y para qué?). Así surgen otras preguntas: ¿'lo que debe ser investigado? ¿lo que debe ser publicado? ¿Quiénes leen los trabajos publicados y para qué sirven?

En el contexto de la producción académica, las motivaciones de los investigadores son regidas por la búsqueda de bonificación (prestigio y diferenciación) que encuentran cabida en la formación de red nacional e internacional, a la vez que son legitimados por las agencias de control y fomento a la investigación, con parámetros dictados por las políticas científicas y tecnológicas e impulsadas por los Estados nacionales pero también por las agencias internacionales.

El marco de esta organización está dada por la competencia entre las áreas de conocimiento, con más o menos legitimidad de unas sobre las demás y donde prevalezcan las tecnociencias en el contexto actual. Por definición, las tecnociencias son las que más se destacan por los usos e incorporación del mercado y son regidas por la constante innovación bajo 
la convocatoria de la sociedad de consumo. En este sentido, hay una colaboración estrecha entre distintas ramas de conocimiento (la mecatrónica, la ingeniería genética o genómica, la biotecnología, la nanotecnología, etc.) integrando de esta manera las investigaciones básicas con las aplicadas.

Sin embargo, es enorme la profusión de nuevas áreas, sub áreas del conocimiento y sus respectivas disciplinas y ni todas se prestan a colaboraciones estrechas o fusiones. Los debates que nascieron a raíz de estas colaboraciones entre distintas áreas y disciplinas han sido realizados con nuevos enfoques, bajo el aporte que algunas investigaciones realizan bajo la óptica de la multi y la interdisciplinariedad.

¿Qué pasa con esas áreas, sub-áreas y disciplinas que no siguen el mismo guión o ideas de las tecnociencias? La crítica que recibe el sistema de ciencias no deriva tan solo de las comunidades académicas, pero son generadas desde una crítica social desde afuera, de los sistemas sociales abiertos. Estas críticas no son puras críticas de manifestaciones callejeras, pero son generadas en los espacios visibles e invisibles de los demás sistemas de conocimiento, de la crítica retórica y semántica, de la resignificación y emergencias de sectores culturales y sociales subalternos. Un buen ejemplo de esto es la disputa de sentidos acerca de las concepciones de desarrollo sustentable por distintos actores: de un lado los hegemónicos y del otro los sectores que funcionan con retóricas de contestación, cómo se dio en el evento de la Cumbre de Rio+20 del 2012.

Las potencialidades de creación de nuevas asociaciones, con un nuevo tipo de crítica hacia las formas tradicionales de producción académica, depende del grado de conflictividad y de competitividad que se establece entre las estructuras de poder epistémico e institucional de las ciencias y las capacidades de generar efectos de contaminación al sistema cartográfico de las ciencias. Un buen ejemplo de esto son los programas de posgrado en medio ambiente y desarrollo en Brasil, desde el 1992 en adelante, lo que produjo efectos en las instituciones nacionales de evaluación y que resultó en la creación de nuevas áreas de conocimiento, reconociéndose la existencia de investigación de carácter interdisciplinar y de un área nueva de ciencias ambientales.

Estas nuevas experiencias derivaron de concepciones distintas de producción académico-científica, más allá de las disciplinas o de concepciones arraigadas de la ciencia positivista, del objetivismo, del elitismo privilegiado del observador, en que los sujetos investigados son tratados con frialdad de un objeto, cuando no con menosprecio, y en muchos casos se les niega su subjetividad, su cultura y sus insustituibles experiencias de prácticas y sus visiones de mundo ${ }^{10}$.

Por fin y en la perspectiva de la ética en la investigación uno debe hacer las siguientes preguntas: ¿Cómo producir una ciencia prudente y pertinente? ¿Cómo establecer vínculos con el contexto (vinculación con el medio)? ¿Una investigación debe hacerse sólo con los objetos aunque es- 
tos son seres humanos? ¿La ciencia beneficia a toda la humanidad? En nombre del progreso ¿es válida toda investigación? ¿De qué ciencia estamos hablando: una ciencia corporativa de investigadores o una ciencia que se hace con todos los demás sujetos y actores involucrados?

Es en este capítulo acribillado de interrogantes que están situadas las discusiones acerca del carácter público de la ciencia, más allá de lo estatal, en que es permitido plantearse otros horizontes para las ciencias, su dimensión política, su pertinencia; para qué y para quiénes se producen los conocimientos en la academia o en otras agencias de conocimiento. En un momento histórico en que las ciencias dominantes son las tecnociencias, las que producen para el mercado y que otras ciencias son controladas por lógicas e intenciones corporativas (una ciencia que se realiza desde la competencia entre los pares y para evaluar y medir la productividad de cada uno), hay que plantearse otros interrogantes que afectarán seguramente nuestras concepciones de ciencia y las formas de cómo nos proponemos a producirla junto a los actores de la ciencia y a los demás. 


\section{Notas}

${ }^{1}$ Según la interpretación de la teoría crítica de la dependencia, la superación del subdesarrollo pasaría por la ruptura de la dependencia y no por la modernización e industrialización de la economía, que al contrario podría incluso incrementar la dependencia. El carácter dramático de la crisis capitalista de la periferia de los años 1960 en adelante impuso a los intelectuales críticos una especie de ultimátum teórico y político: el combate a la dependencia debería implicar la ruptura con el imperialismo e incluso con el capitalismo en si. Pasados ya 50 años, se puede argumentar en favor o en contra de la disyuntiva entre democracia y autoritarismo a pesar de la llegada desde comienzos del 2000 de gobiernos reformadores en algunos países latinoamericanos.

${ }^{2}$ Hay un incontable número de trabajos que desde entonces han sido producidos sobre la época fundacional del pensamiento cepalino y a sus críticos. Consultar entre otros a Furtado (1997), Bielschowsky (1998), Hofman y Torres (2008).

${ }^{3}$ México siempre fue ejemplar en este sentido. La Editorial Fondo de Cultura Económica tenía especialistas en traducción de los clásicos alemanes, por ejemplo; Marx y Weber fueron traducidos desde los años 1950 y eran leídos en toda parte en América Latina, incluso en Brasil.

${ }^{4}$ Para el tema de la colonialidad del saber y del poder, consultar Edgardo Lander (2000), Boaventura de Sousa Santos y Maria Paula Meneses (2010).

5 Las ciencias sociales elijen a sus íconos en cada época. No es distinto con el período contemporáneo en que se nota la influencia de grandes autores en las distintas disciplinas de las ciencias humanas y sociales, tales como Bourdieu, Bauman, Elias, de Sousa Santos, Foucault, Wallerstein, Harvey, etc.

A fin de no dar la impresión de que estamos profesando una sociología de resentimiento intelectual en contra de la teoría y autores considerados una referencia obligatoria, es de fundamental importancia investigar los procesos de ‘derivación' de una situación teórica dada a la creación de un nuevo marco de referencia teórico. Como ejemplo, podríamos tener algunos autores contemporáneos de la teoría sociológica, como Bourdieu (2002), Boltanski (1990), Elias (2005) que buscan reflexionar acerca de su producción teórica (una sociología reflexiva) en el marco de una situación intelectual de su tiempo (Corcuff, 2001). Este ejercicio de producción de una sociología del autor y de su tiempo es posible localizar en Pollak (1996).

${ }^{6}$ El XXX Congreso de ALAS del 29 de noviembre al 4 de diciembre del 2015 en Costa Rica, bajo la llamada 'Pueblos en Movimiento: un nuevo diálogo en las ciencias sociales’ a ejemplo de los últimos congresos de ALAS, presenta 32 Grupos de Trabajo con las problemáticas nuevas de discusión e investigación existentes en las agencias académicas y en el debate social y político actuales (http://www.sociologia-alas.org/pt).

${ }^{7}$ Aquí no hay que confundir el contexto de discusión de los años 1960 (Snow, 1987) entre dos culturas (la científica y la humanística) que eran mutuamente sordas y excluyentes, con lo que es planteado en los años 1990 (Wallerstein et al., 1996) por una epistemología crítica hacia una aproximación entre ciencias humanas, sociales y las demás ciencias naturales y físico-químicas en pos de una práctica interdisciplinaria en la investigación de temas complejos y compartidos, como los estudios en medio ambiente, tecnología, nutrición, salud, etc. 
8 "Comparadas con las sociedades del Norte, las sociedades latinoamericanas no tienen ni "verdaderos" Estados, ni "verdaderas” clases sociales, ni "verdaderas” élites dirigentes, ni "verdaderos" movimientos sociales (...) América Latina no es ni esto ni lo otro. $\mathrm{Ni}$ verdaderamente tradicional ni verdaderamente moderna. En términos analíticos, la temática de las sociedades duales (...) es remplazada por la idea de la dualidad de un lazo social inextricablemente moderno y tradicional; en verdad, ni moderno ni tradicional" (Martucelli, 2010, p. 39).

[9] "Mientras la tradición latinoamericana de análisis social en la segunda mitad del siglo pasado focalizó los procesos de formación de voice, hoy día nos encontramos frente la necesidad creciente de comprender las múltiples dinámicas del exit” ( Sorj y Martuccelli, 2008, p. 15). Los autores se refieren al modelo teórico de Albert Hirschman presentado en su libro Exit, Voice and Loyalty. Cambridge: Harvard UniversityPress, 1970.

${ }^{10}$ En las ciencias sociales, el capítulo de la producción discursiva y el rol del lenguaje sea desde el código de las teorías, de parte del observador calificado, sea de la producción retórica de los actores sociales es todavía un tema abierto a distintos planteos teóricometodológicos y que merecen atención y un seguimiento muy preciso; teorías lingüísticas, sobre las narrativas y los discursos vienen a jugar importante contribución (Bajtin, Wittgenstein, Habermas, Ricoeur, Pecheux, Verón, Laclau, Fairclough...). Acerca de la justificación o de la conciencia que los actores sociales hacen y manifiestan de sus prácticas y auto-interpretaciones, consultar Marx (1981) y Tilly (2006). 
Polis, Revista Latinoamericana, Volumen 14, $N^{\circ}$ 41, 2015

\section{Bibliografía}

Acosta, Alberto (2012), Buen Vivir, SumakKawsay. Una oportunidad para imaginar otros mundos. Abya-Yala, Quito.

Bengoa, José (2007), La emergencia indígena en América Latina, D.F. Fondo de Cultura Económica, México.

Barrington Moore Jr. (1966), Social Origins of Dictatorship and Democracy: Lord and Peasant in the Making of the Modern World. Beacon Press, Boston.

Bellamy Foster, John (2005), A Ecologia de Marx: materialismo e natureza. Civilização Brasileira, Rio de Janeiro.

Bielschowsky, R. (1998) “Evolución de las ideas de la Cepal”. Revista de la CEPAL. Número extraordinario, octubre 1998, Santiago de Chile.

Boltanski, Luc (2000), El amor y la Justicia como competencia. Tres ensayos de sociología de la acción. Amorrortu editores, Buenos Aires.

Bourdieu, Pierre (2002), Esboço de Auto-análise. Zahar, Rio de Janeiro.

Corcuff, Philippe (2001), As Novas Sociologias: construções da realidade social. EDUSC, Bauru.

De La Cuadra, Fernando (2015), “Buen Vivir: ¿Una auténtica alternativa post-capitalista?”, Prólogo. Polis, Revista Latinoamericana, Volumen 14, $\mathrm{N}^{\circ} 40$, p. $7-19$.

Elias, Norbert (2001), Norbert Elias por ele mesmo. Zahar, Rio de Janeiro.

Fanon, Franz (2002), Les damnés de la terre. La Découverte, Paris.

Frank, André Gunder (1969), Latin America: Underdevelopment or Revolution. MonthlyReview Press.

Furtado, Celso (1997), Obra Autobiográfica. Paz e Terra, Rio de Janeiro, 3 v.

Giddens, Anthony y Turner, J. (orgs.) (1999), Teoria Social Hoje. UNESP, São Paulo.

Harvey, David (2005), A Brief History of Neoliberalism. University Press, Oxford.

Ídem (2010.), The Enigma of Capital and the Crises of Capitalism. ProfileBooks.

Hoffman, A. y Torres, M. (2008). “El pensamiento cepalino en la Revista 
de la CEPAL (1976-2008)”. Revista de la CEPAL, n.96, p. 9-26, Santiago do Chile:

LanderR, Edgardo (2000), La colonialidad del saber: eurocentrismo y ciencias sociales. Perspectivaslatinoamericanas. CLACSO, Buenos Aires.

Lipset, Seymour Martin, “Some Social Requisites of Democracy: Economic Development and Political Legitimacy”. American Political Science Review / Volume 53 / Issue 01 / March 1959, pp 69-105.

Marcuse, Herbert (1972), Razón y Revolución. Alianza Editorial, Madrid.

Marini, Rui Mauro (1969), Subdesarrollo y revolución. Siglo XXI, México D.F..

Ídem (1973), Dialéctica de la dependencia. Ediciones Era, México D.F..

Martuccelli, Danilo (2010), ¿Existen individuos en el Sur?, LOM Ediciones, Santiago de Chile.

Marx, Karl (1972), Critique de l'économie politique. Union Générale d’Édition, (Colección 10/18), Paris.

Marx, Carlos y Engels, Federico (1981), El Dieciocho Brumario de Luis Bonaparte. Obras escogidas en tres tomos. Editorial Progreso, tomo I, páginas 404 a 498, Moscú.

Maturana, Humberto y Varela, Francisco (1996), El Árbol del Conocimiento: Las bases biológicas del entendimiento humano. Editorial Universitaria, Santiago de Chile.

Morin, Edgar (1984), Ciencia con Conciencia. Anthropos, Barcelona.

Piaget, Jean (1967), “Nature et méthodes de l'épistémologie”. En Logique et connaissance scientifique, p.3-132. Éditions Gallimard, Paris.

Pollak, Michael (1996), "Max Weber: elementos para uma biografia intelectual”. Mana -Estudos de Antropologia Social, volume 2, número 1, abril de 1996 e volume 2, número 2, outubro de 1996, Rio de Janeiro.

Prigogine, Ilya y Stengeres, Isabelle (1980), A Nova Aliança. Metamorfoses da ciência. UnB, Brasília.

Rostow, Walt Whitman (1960) The stages of economic growth: a noncommunist manifesto. UniversityPress, Cambridge.

Santos, Theotonio dos (1972), Socialismo o Fascismo: el nuevo carácter da dependencia y el dilema latinoamericano. Ed. PLA, Santiago. 
Polis, Revista Latinoamericana, Volumen 14, $N^{\circ}$ 41, 2015

Snow, C.P. (1987),Las dos culturas y un segundo enfoque. Alianza Editorial, Madrid.

Sorj, Bernardo y Martuccelli, Danilo (2008), O Desafio latino-americano: coesão social e democracia. Civilização Brasileira, Rio de Janeiro.

Sousa Santos, Boaventura de, Meneses, Maria Paula (orgs.) (2010), Epistemologias do Sul. Cortez Editora, São Paulo.

Tilly, Charles (2006), Why? What happens when people give reasons...and why. Princeton Univesity Press, Princeton and Oxford.

Wallerstein, Immanuel et al (1996), Para abrir as ciências sociais, Cortez Editor, São Paulo.

Wallersteinm Immanuel (2001), Conocer el Mundo, Saber el Mundo: el fin de lo Aprendido. Una ciencia social para el siglo XXI. Siglo XXI editores, México.

Weber, Max (1965), Essais sur la théorie de la science. Librairie Plon, Paris.

Ídem (2001), La ética protestante y el espíritu del capitalismo. Alianza Editorial, Madrid.

Ziman, John (2000), Real Science: What it is, and what it means. Cambridge University Press.

Recibido: 10.08.2015

Aceptado: 25.08.2015 